(1)

CrossMark

\title{
BioCAST/IFCT-1002: epidemiological and molecular features of lung cancer in never-smokers
}

\author{
Sébastien Couraud ${ }^{1,2,3,4}$, Pierre-Jean Souquet ${ }^{1,2}$, Christophe Paris $^{5}$, Pascal Dô ${ }^{6}$, \\ Hélène Doubre ${ }^{7}$, Eric Pichon ${ }^{8,9}$, Adrien Dixmier ${ }^{10}$, Isabelle Monnet ${ }^{11}$, \\ Bénédicte Etienne-Mastroianni ${ }^{12,13}$, Michel Vincent ${ }^{14}$, Jean Trédaniel ${ }^{15}$, \\ Marielle Perrichon ${ }^{16}$, Pascal Foucher ${ }^{17}$, Bruno Coudert ${ }^{18}$, \\ Denis Moro-Sibilot 19,20, Eric Dansin ${ }^{21}$, Stéphanie Labonne' ${ }^{1,2}$, Pascale Missy ${ }^{22}$, \\ Franck Morin ${ }^{22}$, Hélène Blanché ${ }^{23}$ and Gérard Zalcman ${ }^{24}$, on behalf of The \\ French Cooperative Intergroup IFCT ${ }^{25}$
}

Affiliations: ${ }^{1}$ Dept of Respiratory Medicine, Lyon Sud Hospital, Hospices Civils de Lyon, Lyon, France. ${ }^{2}$ Faculty of Medicine Lyon-Sud Charles Mérieux, Lyon 1 University, Lyon, France. ${ }^{3}$ Centre for Clinical Epidemiology, Lady Davis Institute for Medical Research, Jewish General Hospital, Montreal, Quebec, Canada. ${ }^{4}$ Dept of Epidemiology, Biostatistics and Occupational Health, McGill University, Montreal, Quebec, Canada. ${ }^{5}$ (CP) INGRES, EA7298, Lorraine University, Vandoeuvre Les Nancy, France. ${ }^{6}$ Francois Baclesse Cancer Institute, Caen, France. ${ }^{7}$ Pulmonology Unit, Foch General Hospital, Paris, France. ${ }^{8}$ Pulmonology Unit, University Hospital of Tours, Tours, France. ${ }^{9}$ François Rabelais University, Respiratory Diseases Study Centre, U1100/ EA6305, Tours, France. ${ }^{10}$ Pulmonology Únit, Orleans Regional Hospital, Orléans, France. ${ }^{11}$ Pulmonology Unit, Centre Hospitalier Intercommunal de Créteil, Créteil, France. ${ }^{12}$ Dept of Respiratory Medicine, Hôpital Louis Pradel, Hospices Civils de Lyon, Lyon, France. ${ }^{13}$ Claude Bernard University Lyon 1, Lyon, France. ${ }^{14}$ Pulmonology Dept, Saint Joseph-Saint Luc Hospital, Lyon, France. ${ }^{15}$ Pulmonology Dept, Paris - Saint Joseph Hospital, and Sorbonne Paris Cité University, Paris, France. ${ }^{16}$ Pulmonology Dept, Bourg-en-Bresse General Hospital, Bourg en Bresse, France. ${ }^{17}$ Thoracic Oncology, Dijon University Hospital, Dijon, France. ${ }^{18}$ Oncology Dept, Georges François Leclerc Cancer Centre, Dijon, France. ${ }^{19}$ Pulmonology and Thoracic Oncology Dept, Grenoble University Hospital, Grenoble, France. ${ }^{20}$ Medicine Faculty, Joseph Fourrier University, La Tronche, France. ${ }^{21}$ Oncology Dept, Oscar Lambret Cancer Centre, Lille, France. ${ }^{22}$ Intergroupe Francophone de Cancérologie Thoracique, Paris, France. ${ }^{23}$ Fondation Jean Dausset - CEPH, Paris, France. ${ }^{24}$ Pulmonology and Thoracic Oncology Dept, Caen University Hospital, and UMR INSERM 1086, Caen BasseNormandie University, Caen, France. ${ }^{25}$ For collaborators of the BioCAST/IFCT-1002 study, please see the acknowledgements section.

Correspondence: Sébastien Couraud, Service de Pneumologie, Hospices Civils de Lyon, CH Lyon Sud, 165 Chemin du Grand Revoyet, F-69495 Pierre Bénite, France. E-mail: sebastien.courauddachu-lyon.fr

ABSTRACT Lung cancer in never-smokers (LCINS) (fewer than 100 cigarettes in lifetime) is considered as a distinct entity and harbours an original molecular profile. However, the epidemiological and molecular features of LCINS in Europe remain poorly understood.

All consecutive newly diagnosed LCINS patients were included in this prospective observational study by 75 participating centres during a 14-month period. Each patient completed a detailed questionnaire about risk factor exposure. Biomarker and pathological analyses were also collected. We report the main descriptive overall results with a focus on sex differences.

384 patients were included: 65 men and 319 women. 66\% had been exposed to passive smoking (significantly higher among women). Definite exposure to main occupational carcinogens was significantly higher in men (35\% versus $8 \%$ in women). A targetable molecular alteration was found in $73 \%$ of patients (without any significant sex difference): EGFR in 51\%, ALK in $8 \%, K R A S$ in $6 \%, H E R 2$ in $3 \%, B R A F$ in $3 \%, P I 3 K C A$ in less than $1 \%$, and multiple in $2 \%$.

We present the largest and most comprehensive LCINS analysis in a European population. Physicians should track occupational exposure in men (35\%), and a somatic molecular alteration in both sexes (73\%).

@ERSpublications

Occupational exposure and targetable mutation should be tracked in lung cancer of European never-smokers. http://ow.ly/FB2WS 


\section{Introduction}

Although tobacco smoking is the strongest causal factor for lung cancer, $10-25 \%$ of lung cancer worldwide occurs in lifelong nonsmokers (300000 deaths each year) [1-3]. A "never-smoker" is well-defined as an individual who has smoked fewer than 100 cigarettes during their lifetime. Lung cancer occurring in never-smokers (LCINS) is now considered a distinct entity. However, this disease appears strongly linked to geographical origins. For example, LCINS is drastically more common in Asia than in the USA or Europe [2]. This could be related to distinct features pertaining to inherited susceptibility, as well as to varied exposure to occupational and environmental carcinogens in different geographical areas. Thus, it is possible that LCINS is, in fact, globally a very heterogeneous disease $[1,2]$. Although the disease is thought to be well characterised, very little data on LCINS are available in Europe [4], and nobody has concomitantly assessed most of the known risk factors for lung cancer.

LCINS is also known to hold an original spectrum of driver mutations. Thus, human epidermal growth factor receptor family (EGFR (also called HER1) and HER2 (human epidermal growth factor receptor 2)) mutations appear more frequent, while KRAS (Kirsten rat sarcoma viral oncogene homologue) and $B R A F$ ( $\mathrm{v}$-Raf murine sarcoma viral oncogene homologue B), thought to be related to tobacco carcinogens, are less common in this group [5-7]. Finally, $A L K$ (anaplastic lymphoma kinase) rearrangements also occur slightly more frequently in never-smoking patients [8]. This spectrum is another argument favouring the hypothesis that separate genetic pathways lead to lung carcinogenesis in ever- and never-smokers [1].

Given that the clinical and molecular epidemiology of LCINS in Europe is still poorly understood, the French Collaborative Intergroup for Thoracic Cancer Research (IFCT) sought to investigate this specific entity through a prospective cohort of LCINS. The BioCAST (IFCT-1002) study's main objective was to describe a French population of LCINS patients and especially the distribution of risk factor exposure and biomarker patterns. Here we report this study's main descriptive results, focusing on potential sex differences among LCINS patients.

\section{Population and methods}

\section{Population}

Main inclusion criteria were: 1) being a self-declared never-smoker (i.e., declaring having smoked fewer than 100 cigarettes during lifetime); 2) being newly diagnosed with a non-small cell lung cancer (NSCLC) by biopsy or by cytological sampling; 3) being aged at least 18 years; 4) speaking and understanding French (or having access to a relative able to translate); 5) having phone access, either at home or at the hospital; and 6) granting signed consent.

The IFCT sponsored the BioCAST study. The study was conducted in 75 participating centres throughout metropolitan France, from November 1, 2011 to January 31, 2013. Participating centres were asked to include all consecutive newly diagnosed LCINS cases. IFCT research staff members were in charge of administrative management and quality assurance (in compliance with international research standards) [9].

\section{Ethics}

The Sud-Est IV Lyon ethics committee approved the study protocol on September 13, 2011. The Advisory Committee on Information Processing for Health Research permitted use of a computerised database on September 8, 2011, and the National Commission for Data Protection was contacted on September 23, 2011, in accordance with French law. Blood sample collection was declared to the French Ministry of Research on July 1, 2011. The BioCAST study was registered on the US National Institute of Health website www.clinicaltrials.gov under the CTC ID NCT01465854.

\section{Study design}

Patients signed their consent after receiving information about the study from their physicians. Afterward, and before any anti-cancer treatment, each patient's blood was sampled. Patients were then contacted by phone in order to schedule a dedicated phone interview and complete a standardised questionnaire. All

For editorial comment see Eur Respir J 2015; 45: 1214-1217 [DOI: 10.1183/09031936.00046915]

This article has supplementary material available from erj.ersjournals.com.

Received: March 52014 | Accepted after revision: Nov 232014 | First published online: Feb 052015

Support statement: The BioCAST/IFCT-1002 study was supported by research grants from Astra-Zeneca, Boehringer Ingelheim, Lilly, Pfizer, Pierre-Fabre and Roche. Funding for this article has been deposited with FundRef. Those awarding funding had no role in the design, analysis and interpretation of the results, and the authors worked independently.

Conflict of interest: Disclosures can be found alongside the online version of this article at erj.ersjournals.com 
patients had the opportunity to fully prepare this questionnaire before the pre-planned interview. Patients were also encouraged to seek assistance from a relative if needed (in cases of fatigue, deafness, memory deficiency or poor spoken French). Two trained research assistants performed all the interviews in order to control for reporting and interrogation biases. Additional medical data, as well as molecular testing and pathological reports were collected directly from participating physicians.

\section{Patients' questionnaire}

A 17-page questionnaire was delivered to patients upon inclusion. It included questions about demographics, socio-educative level and alternative tobacco consumption, as well as cannabis smoking, passive smoking exposure, occupational exposure, personal medical history, family history, alcohol intake and fried and stir-fried cooking exposure. Occupational exposures to bronchial carcinogens were assessed using a lifelong task-based questionnaire, the efficacy of which for detecting occupational exposure was recently published [10]. All patients were questioned about their home addresses and exposure to solid fuel for cooking or heating. Finally, women declared oral contraceptive and post-menopausal hormone replacement therapy intake, as well as other details on reproductive factors. Additional detailed information on recorded data and exposure measurements are provided in supplemental file $\mathrm{S} 1$.

\section{Biomarker analyses}

The French National Cancer Institute (INCa) launched a network of 28 molecular genetics platforms that provide routine cancer molecular testing for all patients [11]. Each BioCAST participant centre was asked to systematically order tests for somatic mutations in EGFR and KRAS, as well as ALK fusion gene, to its local molecular genetics laboratory. Investigator sites were also encouraged to request $B R A F, H E R 2$, and PI3KCA mutation analyses, which are also routinely performed (free of charge to the patient) at these platforms. All centres were advised to follow local policy, and were therefore allowed to forego further mutation testing if one mutation reputed to exclude the others was found. Final and detailed reports of these analyses were collected for each patient.

Actually, biomarker testing methods are not homogeneous throughout all centres. However, the ERMETIC study was designed to assess concordance of results between each centres (blinded cross validation study compared to an international reference lab) and between the different methods used in such centres (direct sequencing, PCR-based, Restriction fragment-length polymorphism, and high-resolution melting). This validation study showed good concordance rate suggesting that, despite some difference in sample processing and analyses, results are accurate $[12,13]$.

\section{Blood samples bio-bank}

Four tubes of each patient's blood were collected for further studies (two stored in EDTA, one dry and one ACD-citrated). Samples were transported to the BioCAST central laboratory (hosted at the Centre d'Étude du Polymorphisme Humain, Fondation Jean Dausset, Paris, France) at room temperature within $24 \mathrm{~h}$ of sampling and were then processed.

\section{Statistics}

Categorical variables were expressed as percentages. Comparisons of proportions used the Chi-squared test when the expected count in a given category was at least five, or Fisher's exact test otherwise. We used the one-sample Kolmogorov-Smirnov test in order to assess the plausibility of a normal distribution assumption for continuous variables. Normally distributed continuous variables were expressed as mean and standard deviation. Comparisons of means were conducted using the bilateral t-test. Differences in distribution of continuous variables between two independent samples were assessed using the Mann-Whitney U-test, and the Kruskal-Wallis one-way ANOVA was used to compare more than two independent samples. Some continuous variables were also categorised in quartiles, tertiles or clinically relevant categories according to their distribution in the overall population. Missing values were reported as such, and all tests were two-sided. All statistics were calculated using the SPSSv20 (IBM SPSS Statistics, New York, NY, USA).

\section{Results}

Population

Altogether, 384 consecutive French never-smokers were included during the study period. Of these, 336 (87.5\%) completed the interview, 359 (93\%) had at least one biomarker testing, and 381 (99.2\%) underwent the correct blood sampling procedure (fig. 1).

\section{Main demographic data}

Table 1 gives the overall population's main features and a sex comparison. The sex ratio was 4.9 in favour of women. There was no patient-reported alternative smoking (water-pipe, cigar, pipe or gum) or cannabis 


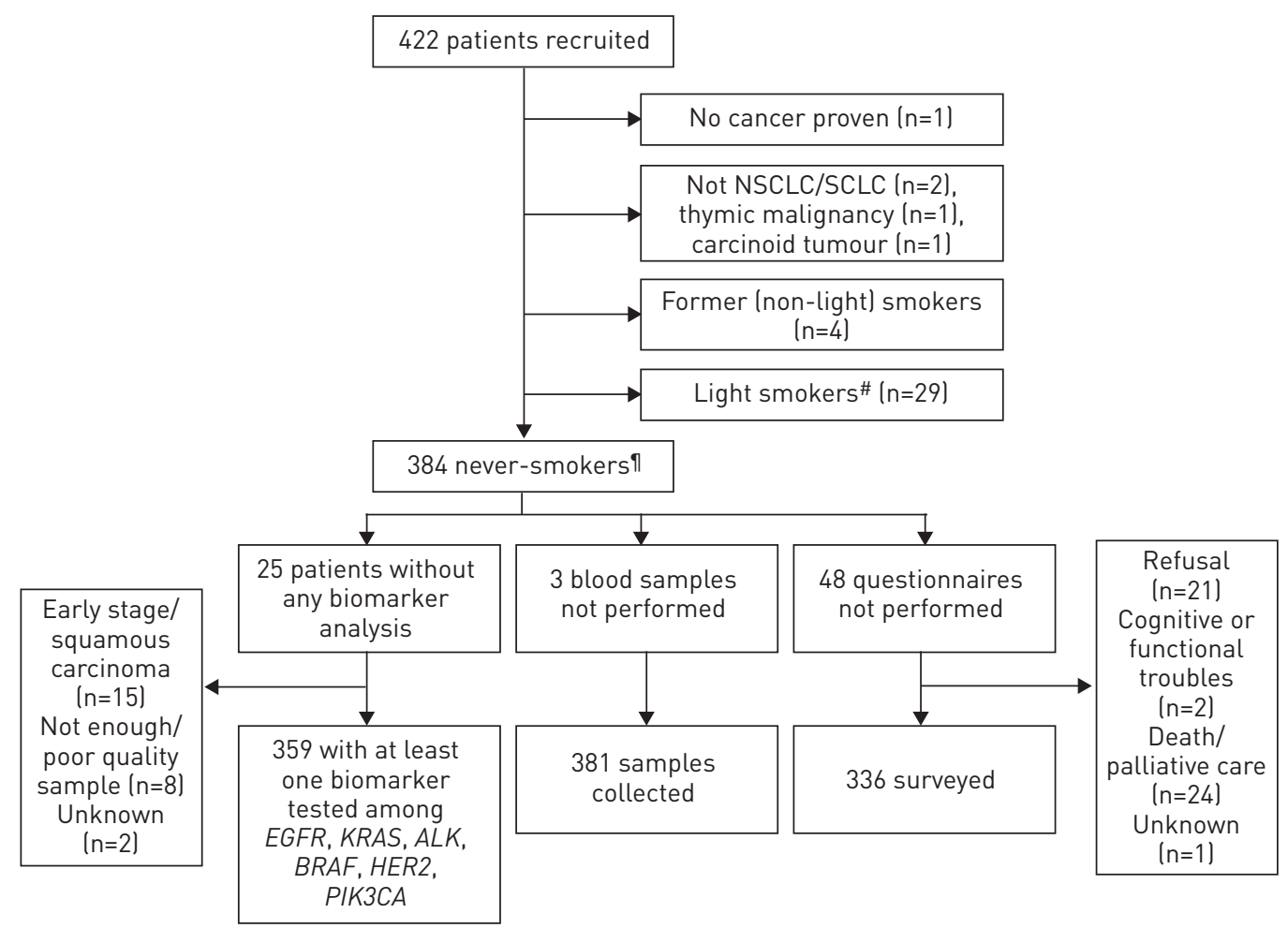

FIGURE 1 Flow chart of the BioCAST study. NSCLC: nonsmall cell lung cancer; SCLC: small cell lung cancer; EGFR: epidermal growth factor receptor; KRAS: Kirsten rat sarcoma viral oncogene homologue; $A L K$ : anaplastic lymphoma kinase; $B R A F$ : v-Raf murine sarcoma viral oncogene homologue B; HER2: human epidermal growth factor receptor 2; PIK3CA: phosphatidylinositol-3-kinase, catalytic subunit $\alpha .{ }^{*}$ : smoked fewer than 5 packs.year $^{-1}$ in lifetime and quit at least 15 years prior; ": smoked fewer than 100 cigarettes in lifetime.

use. $91 \%$ of patients originated from Europe: 276 from Western Europe, 22 from Southern Europe and six from Eastern Europe. Men were, on average, 4 years younger than women $(p=0.016)$, and the proportion of patients aged $<55$ years old at diagnosis was significantly higher among males. In addition, men were better educated, more pre-obese and exhibited higher alcohol consumption than women.

\section{Histology and stage of the disease}

The main pathological lung cancer features found in our 384 patients are shown in table S2 in the online supplementary material. We found no significant difference between the sexes. Notably, the frequency of squamous cell carcinoma (SCC) was similar in both sexes ( $9 \%$ in women versus $7 \%$ in men; $\mathrm{p}=0.606$ ). Most cases were adenocarcinoma (85\%). The other histological types were SCC (8\%), large cell carcinoma $(4 \%)$, adenosquamous $(n=5)$, sarcomatoid carcinoma $(n=4)$ and carcinoma not otherwise specified $(n=2)$. thyroid transcription factor 1 (TTF-1) immunostaining was positive in $76 \%$ of patients. Table S3 in the online supplementary material shows sample type and origin. Most were core biopsies (90\%) from primitive tumours (69\%) and were classified as stage IV (73\%).

\section{Occupational exposure}

Data about exposure to occupational carcinogens was available in 334 patients, and results are presented in table 2. Altogether, $13 \%$ of patients were found to have been definitely exposed to at least one occupational carcinogen, with a striking difference between the sexes: $35 \%$ of men compared with only $8 \%$ of women $\left(\mathrm{p}<10^{-4}\right)$. The most frequent carcinogenic agent was polycyclic aromatic hydrocarbons, followed by asbestos, silica and diesel exhaust. Whatever the agent considered, men were in all cases significantly more exposed than women.

\section{Passive smoke exposure}

Overall, 219 (66\%) of the 334 patients were ever exposed to passive smoking, either in a domestic setting (59\%) or in the workplace (18\%) (table 3). Domestic exposure to passive smoking was significantly more 
TABLE 1 Main characteristics of the BioCAST population

\begin{tabular}{|c|c|c|c|c|c|}
\hline & \multicolumn{4}{|c|}{ Lung cancer in never smokers (BioCAST) } & \multirow[t]{2}{*}{ Lung cancer in ever smokers } \\
\hline & Men & Women & p-value & All & \\
\hline Total patients $\mathbf{n}$ & & & & 384 & 6246 \\
\hline Women & & 319 (83) & & & 19 \\
\hline Men & 65 (17) & & & & 81 \\
\hline Age & $66.6 \pm 13.0$ & $70.5 \pm 11.7$ & $0.016^{\pi}$ & $69.8 \pm 12.0$ & $65.5 \pm 11.3^{+}$ \\
\hline Missing data $n$ & 7 & 41 & & 48 & \\
\hline Europe & $51 / 58(88)$ & $253 / 278(91)$ & $0.468^{\S}$ & $304 / 336(91)$ & \\
\hline Africa & 4/58 (7) & $12 / 278(4)$ & & $16 / 336(5)$ & \\
\hline Asia & $1 / 58(2)$ & $9 / 278(3)$ & & $10 / 336(3)$ & \\
\hline Caribbean & $2 / 58(3)$ & $4 / 278(1)$ & & $6 / 336(2)$ & \\
\hline \multicolumn{6}{|l|}{ Education level } \\
\hline Body mass index $\mathrm{kg} \cdot \mathrm{m}^{-2}$ & $25.4 \pm 4.8$ & $23.7 \pm 5.4$ & $0.015^{f}$ & $24.2 \pm 5.5$ & \\
\hline Missing data $\mathrm{n}$ & 7 & 46 & & 53 & \\
\hline Underweight/normal & $27 / 58(47)$ & $165 / 273(60)$ & 0.087 & $192 / 331(58)$ & \\
\hline Pre-obese & $24 / 58(41)$ & $74 / 273$ (27) & & $98 / 331(30)$ & \\
\hline Obese & $7 / 58(12)$ & $34 / 273(13)$ & & $41 / 331(12)$ & \\
\hline \multicolumn{6}{|c|}{ Alcohol intake (in standard glass per day) } \\
\hline Missing data $\mathrm{n}$ & 7 & 43 & & 50 & \\
\hline $0-1$ & 49/58 (85) & $265 / 276(96)$ & $0.003^{\# \#}$ & $314 / 334(94)$ & \\
\hline$\geqslant 2$ & $9 / 58(16)$ & $11 / 276(4)$ & & $20 / 334(6)$ & \\
\hline
\end{tabular}

Data are presented as $\mathrm{n}(\%)$, median \pm interquartile range or $\mathrm{n} / \mathrm{N}$ available $(\%)$, unless otherwise stated. ${ }^{\#}$ : data from the KBP-CPHG study [14]; ": t-test; ${ }^{+}$: both smokers and never smokers ( $\mathrm{n}=7051$ ); not available for smokers only; ${ }^{\S}$ : computed between "European" and "non-European" categories; ${ }^{f}$ : Mann-Whitney $U$ test; ${ }^{\# \#}$ : Fisher's exact test; all others are Chi-squared tests (for categorical variables).

common among women than men (64 versus 38\%; $\mathrm{p}=0.0001$ ), but this sex difference was not observed for workplace exposure. Moreover, of those exposed in a domestic setting, women were exposed for a significantly longer period than men. For patients exposed in a domestic setting, exposure began during childhood in $62 \%$ of patients. Men were more frequently exposed in childhood than women, suggesting that women were mainly exposed via their spouses.

Domestic pollution

Table S4 in the online supplementary material provides exposure to domestic pollution. Women more frequently reported having ever been exposed to cooking oil fumes (41 versus 18\%; $\mathrm{p}=0.001$ ). $26 \%$ of

TABLE 2 Definite exposure to occupational carcinogens (by task) according to sex

\begin{tabular}{lcccc} 
& Men & Women & p-value & All \\
\hline Overall lat least one) & $20(35)$ & $23(8)$ & $<10^{-4}$ & $43(13)$ \\
PAH & $15(26)$ & $15(5)$ & $<10^{-4}$ & $30(9)$ \\
Asbestos & $11(19)$ & $11(4)$ & $<10^{-4 \#}$ & $22(7)$ \\
Silica & $10(18)$ & $5(2)$ & $<10^{-4 \#}$ & $15(4)$ \\
Diesel & $6(11)$ & $2(1)$ & $<10^{-4 \#}$ & $8(2)$ \\
Chrome & $5(9)$ & $1(0)$ & $0.001^{\#}$ & $6(2)$ \\
Paint & $4(7)$ & $3(1)$ & $0.018^{\#}$ & $7(2)$ \\
Missing data & 8 & 42 & & 50 \\
\hline
\end{tabular}

Data are presented as $\mathrm{n}(\%)$, unless otherwise stated. PAH: polycyclic aromatic hydrocarbons. ${ }^{\text {: }}$. Fisher's exact test, others are Chi-squared tests. 
TABLE 3 Passive smoking exposure according to sex

\begin{tabular}{|c|c|c|c|c|c|c|c|c|c|c|c|c|}
\hline & \multicolumn{4}{|c|}{ Domestic exposure } & \multicolumn{4}{|c|}{ Workplace } & \multicolumn{4}{|c|}{ Overall } \\
\hline & Men & Women & p-value & All & Men & Women & $\mathrm{p}$-value & All & Men & Women & p-value & All \\
\hline Missing $\mathbf{n}$ & 7 & 43 & & 50 & 7 & 43 & & 50 & 7 & 43 & & 50 \\
\hline Never exposed & $36 / 58(62)$ & $100 / 276(36)$ & $<10^{-4}$ & $136 / 334(41)$ & $47 / 58(81)$ & $227 / 276$ (82) & 0.827 & $274 / 334$ (82) & $30 / 58(52)$ & $85 / 276(31)$ & 0.002 & $115 / 334(34)$ \\
\hline Ever exposed & $22 / 58(38)$ & $176 / 276(64)$ & & 198/334 (59) & $11 / 58(19)$ & $49 / 276(18)$ & & $60 / 334(18)$ & $28 / 58$ (48) & $191 / 276$ (69) & & $219 / 334(66)$ \\
\hline Missing data & & 4 & & 4 & & 1 & & 1 & & 3 & & 3 \\
\hline \multicolumn{13}{|l|}{ Length of exposure } \\
\hline$<20$ years & $14 / 22(64)$ & 59/172 (34) & 0.008 & 73/194 (38) & $8 / 11(73)$ & $23 / 48$ (48) & NC & $31 / 59(53)$ & $15 / 28(54)$ & 49/188 (26) & 0.002 & $64 / 216(30)$ \\
\hline $20-30$ years & $7 / 22$ (32) & $57 / 172(33)$ & & $64 / 194$ (33) & $2 / 11(18)$ & $15 / 48(31)$ & & $17 / 59(29)$ & $11 / 28(39)$ & $70 / 188$ (37) & & $81 / 216(38)$ \\
\hline$>30$ years & $1 / 22(5)$ & $56 / 172(33)$ & & $57 / 194(29)$ & $1 / 11(9)$ & $10 / 48(21)$ & & $11 / 59(19)$ & $2 / 28(7)$ & $69 / 188(37)$ & & $71 / 216$ (33) \\
\hline Exposed in childhood & $18 / 22(82)$ & 104/172 (59) & 0.039 & $122 / 194(62)$ & & & & & & & & \\
\hline Exposed in adulthood only & $4 / 22(8)$ & $72 / 172(41)$ & & $76 / 194(38)$ & & & & & & & & \\
\hline
\end{tabular}


patients reported to have been exposed for $>50 \%$ of their lifetimes to solid fuel fumes; here there was no significant difference between men and women.

\section{Personal and familial medical history}

$24 \%$ of patients reported having at least two biological first-degree relatives with lung cancer, and $17 \%$ reported a personal history of at least one other cancer. Medical histories of pertussis, tuberculosis and pneumonia were reported in $21 \%, 8 \%$ and $6 \%$ of patients, respectively. In addition, $13 \%$ had been diagnosed with a chronic bronchial disease. There were no differences between the sexes with respect to these variables (Table S5 in the online supplementary material).

Reproductive factors and hormone intake in women are reported in table S6 in the online supplementary material. Overall, 115 (42\%) patients had used oral contraceptives, and 70 (25\%) had undergone post-menopause hormonal replacement therapy.

\section{Biomarkers (somatic mutations)}

EGFR mutations were tested in 340 patients, KRAS in 293, ALK rearrangements in 192, BRAF in 22, HER2 in 201, and PI3KCA in 187. Altogether, we found 220 molecular alterations in 208 patients (table 4). Six patients carried a somatic mutation within two $(n=5)$ or three $(n=1)$ genes simultaneously (multiple mutations), while five others hosted two simultaneous somatic mutations in the EGFR gene (table S7 in the online supplementary material). Alterations in the EGFR gene were the most common: 147 patients displayed 153 mutations, with deletions in exon 19 and substitution L858R in exon 21 being the most frequent ( $\mathrm{n}=74$ and 33 , respectively). We also found one missense mutation T790M in exon 20 and one alteration never reported before in the Catalogue of Somatic Mutations in Cancer (COSMIC) database (http://cancer.sanger.ac.uk/cancergenome/projects/cosmic) (c.2303_2305delinsTCT in exon 20). In addition, 24 gene fusions involving the ALK gene were observed in 23 patients. We also noted 20 KRAS mutations in 18 patients, 10 BRAF mutations in nine patients, eight HER2 mutations in eight patients, and five PI3KCA mutations in three patients. Overall, an EGFR mutation was found in $43 \%$ of individuals, a KRAS mutation in 7\%, a BRAF mutation in 5\%, a HER2 mutation in $4 \%$, a PIK3CA (phosphatidylinositol3-kinase, catalytic subunit $\alpha$ ) mutation in $2 \%$, and an $A L K$ rearrangement in $13 \%$ of patients tested for the corresponding biomarker. We found no significant difference in the mutation frequency as well as in the mutation type according to the gender. For EGFR, women exhibit a higher frequency than men but the difference remains non-significant (39 versus $44 \%$ respectively; $\mathrm{p}=0.438$ ). In addition, we found no difference among sex for the type of mutation found in each biomarker; but however, women tend to exhibit more KRAS transition mutations than men (table 5).

Taking all these data together, 77 (27\%) patients were considered "pan-negative" (all biomarkers found were wild type; or at least wild-type EGFR, KRAS and ALK simultaneously). In the remaining 284 patients with complete data, the most common alteration found was an EGFR mutation (in $51 \%$ of patients), followed by an ALK rearrangement (8\%), a missense mutation in KRAS (6\%), HER2 (3\%), BRAF (3\%) and PIK3CA (<1\%). Only $2 \%$ carried multiple mutations (see fig. 2). Therefore, $73 \%$ of French never-smokers carried a targetable molecular alteration.

\section{Discussion}

Among French never-smokers with lung cancer, we found that occupational exposure to carcinogens was significantly higher in men than in women, whereas domestic exposure, both to passive smoking and to cooking-oil, was higher in women. Most of the tumours we studied (73\%) carried a targetable mutation.

To our knowledge, our study is the largest and most comprehensive ever conducted that focused on LCINS in a European population. Indeed, most published cohort studies used a retrospective design, were dedicated to Asian populations and/or explored only a single or a small subset of risk factors simultaneously. In Europe, the EPIC (European Prospective Investigation into Cancer and Nutrition) cohort hosted 97 newly diagnosed lung cancer cases [15]. In Asia, a paper from KIM et al. [16], recently reported to be the largest and most comprehensive series on never-smokers, effectively analysed 229 Asian patients, using a retrospective review for EGFR, KRAS and ALK. KAWAGUSH et al. [17] also keep a prospective registry of never-smoker from Japan, Singapore, Korea as well as few patients in US, but they only reported their results on passive smoking exposure.

Our main findings are consistent with published literature. In fact, CLÉMENT-DuCHÊNE et al. [18] have already shown, in 67 French never-smokers, that occupational exposure and passive smoking were differently distributed between the sexes. Indeed, some lung cancers are known to be related to domestic pollution exposure, although modestly, even in Europe [19]. Regarding biomarker distribution, we have shown that French never-smokers carry $73 \%$ of targetable mutations. This finding situates French never-smoker between Asian (more than 80\%) and American (55\%) never-smokers [1, 20, 21]. 


\section{TABLE 4 Mutation patterns observed in the BioCAST study}

Mutation by biomarker and exon

\begin{tabular}{ccccc}
$\begin{array}{c}\text { Number of mutations in the } \\
\text { same patient }\end{array}$ & Total $n$ & $\%$ of gene & $\%$ of total \\
\hline One $\quad$ Two & Three &
\end{tabular}

BRAF

Exon 15

V600E

L597L

Total

7

1

1

9

Exon 18

G719C

G719A

Unspecified

Total

Exon 19

Deletion

Unspecified

Total

Exon 20

c.2303 2305delinsTCT

T790M

S768I

Insertion

Duplication

Deletion

Unspecified

Total

Exon 21

L858R

L861Q

Unspecified substitution

P848L

Unspecified

Total

Unspecified

Total

Total

PI3KCA

Exon 9

E545K

Unspecified substitution

Unspecified

Exon 20

Unspecified

Total

HER2

Exon 20

Unspecified substitution

Unspecified insertion

Unspecified duplication

Total

KRAS

Exon 2

G13D

G12V

G12R

G12G

G12D

G12C

G12A

Unspecified

Total

ALK

Unspecified

Total

Total

$\begin{array}{ll}1 & 1 \\ 1 & 1 \\ 1 & 1\end{array}$

1
10

$5 \%$

$74 \quad 2$

276

$12 \quad 12$

$88-58 \%$

$1-1$

$\begin{array}{lll}1 & 1 & 1 \\ 4 & 1 & 1 \\ 1 & & 1 \\ 1 & & 1 \\ & 1 & 1\end{array}$

$3 \%$

$8 \%$

$33 \quad 36$

$\begin{array}{lll}4 & 1 & 36\end{array}$

4

1

1

$32 \%$

$1 \%$

$\begin{array}{lll}1 & 1 \% & \mathbf{7 0} \%\end{array}$

1

$80 \%$

1

1

$20 \%$

$2 \%$

$4 \%$

BRAF: v-Raf murine sarcoma viral oncogene homologue B; EGFR: epidermal growth factor receptor; PIK3CA: phosphatidylinositol-3-kinase, catalytic subunit $\alpha ; H E R 2$ : human epidermal growth factor receptor 2; KRAS: Kirsten rat sarcoma viral oncogene homologue; $A L K$ : anaplastic lymphoma kinase. 
TABLE 5 Mutation profile for each biomarker according to sex

\begin{tabular}{|c|c|c|c|c|c|}
\hline & \multicolumn{4}{|c|}{ Lung cancer in never smokers (BioCAST) } & \multirow[t]{2}{*}{ Lung cancer in ever smokers } \\
\hline & Men & Women & p-value & All & \\
\hline Total $\mathbf{n}$ & & & & 384 & 7789 \\
\hline \multicolumn{6}{|l|}{ EGFR } \\
\hline Patients $n$ & & & & 340 & \\
\hline Wild type & $35(61)$ & $158(56)$ & 0.438 & 193 (57) & \\
\hline Mutation & $22(39)$ & $125(44)$ & & $147(43)$ & $5 \%$ \\
\hline Data missing & 1 & 6 & & 7 & \\
\hline in exon 18 & $1 / 21(5)$ & $2 / 119(2)$ & NC & $3 / 140(2)$ & \\
\hline in exon 19 & $12 / 21(57)$ & $73 / 119(61)$ & & $85 / 140(61)$ & \\
\hline in exon 20 & 0 & $7 / 119(6)$ & & $7 / 140(5)$ & \\
\hline in exon 21 & $8 / 21(38)$ & $37 / 119(31)$ & & $45 / 140(32)$ & \\
\hline \multicolumn{6}{|l|}{ KRAS } \\
\hline Patients n & & & & 293 & \\
\hline Wild type & $50(94)$ & 223 (93) & $1.0^{\pi}$ & 273 (93) & \\
\hline Mutation & $3(6)$ & $17(7)$ & & $20(7)$ & $32 \%$ \\
\hline Data missing & & 2 & & 2 & \\
\hline Transition & 0 & $6 / 15(40)$ & $0.515^{\pi}$ & $6 / 18(33)$ & \\
\hline Transversion & $3 / 3(100)$ & $9 / 15(60)$ & & $12 / 18(67)$ & \\
\hline \multicolumn{6}{|l|}{$A L K$} \\
\hline Patients $\mathrm{n}$ & & & & 192 & \\
\hline Wild type & $29(94)$ & $139(86)$ & $0.379^{\pi}$ & $168(88)$ & \\
\hline Mutation & $2(6)$ & $22(14)$ & & $24(13)$ & $4 \%$ \\
\hline \multicolumn{6}{|l|}{ BRAF } \\
\hline Patients $\mathrm{n}$ & & & & 222 & \\
\hline Wild type & 34 (94) & $178(96)$ & $0.667^{\pi}$ & 212 (95) & \\
\hline Mutation & $2(6)$ & $8(4)$ & & $10(5)$ & $2 \%$ \\
\hline \multicolumn{6}{|l|}{ HER2 } \\
\hline Patients n & & & & 201 & \\
\hline Wild type & $32(94)$ & $161(96)$ & $0.625^{\pi}$ & $193(96)$ & \\
\hline Mutation & $2(6)$ & $6(4)$ & & $8(4)$ & $<1 \%$ \\
\hline \multicolumn{6}{|l|}{ PIЗKCA } \\
\hline Patients n & & & & 187 & \\
\hline Wild type & $30(97)$ & $153(98)$ & $0.519^{\pi}$ & 183 (98) & \\
\hline Mutation & $1(3)$ & $3(2)$ & & $4(2)$ & $2 \%$ \\
\hline
\end{tabular}

Data are presented as $\mathrm{n}(\%)$ or $\mathrm{n} / \mathrm{N}$ available (\%), unless otherwise stated. EGFR: epidermal growth factor receptor; KRAS: Kirsten rat sarcoma viral oncogene homologue; ALK: anaplastic lymphoma kinase; BRAF: v-Raf murine sarcoma viral oncogene homologue B; HER2: human epidermal growth factor receptor 2; PIK3CA: phosphatidylinositol-3-kinase, catalytic subunit $\alpha$. \#: Data from Biomarker France [22]; ๆ: Fisher's exact test; all other are Chi-squared test.

Our study does carry some limitations. First, due to financial constraints, we had no control patients. Thus, our cohort could not be used in order to estimate any risk ratios. However, our only aim was to assess the prevalence of known lung cancer risk factors and explore their actual distribution among certain subgroups. In order to put our findings into perspective, our data were compared to those of two recently published, nationwide epidemiological studies assessing the main features of French lung cancer smoker patients: 1) the KBP-CPHG (Cancer broncho-pulmonaire du Collège de Pneumologie des Hôpitaux Généraux) 2010 study, which includes 6246 lung cancers in smokers, for demographical, histological and staging data [14] (table 1);2) the "Biomarker-France" study, sponsored by IFCT and funded by French NCI (INCa), of which preliminary results were reported after inclusion of the first 7789 lung cancers from smokers, for mutation profile data [22] (table 5). Indeed, our data perfectly mirror both the clinical and biological features of ever-smoker lung cancer patients, as reported in those two large series, conducted in the same French background, during the same period of time as BioCAST [14]. However, most of the collected variables had never been assessed in comparable epidemiological studies, or according to smoking status and, thus, are lacking of any comparable data in independent series of patients. Besides, our findings were congruous with existing literature: lung cancer in never smoker is more frequent among women, and adenocarcinoma. In addition, the mutation profile in never smoker strongly differs from smokers [1]. A second critical point is the retrospective reporting of risk factor exposures by patients themselves, without any possibility for a biological exposure objective assessment. However, we tried to minimise interrogation 


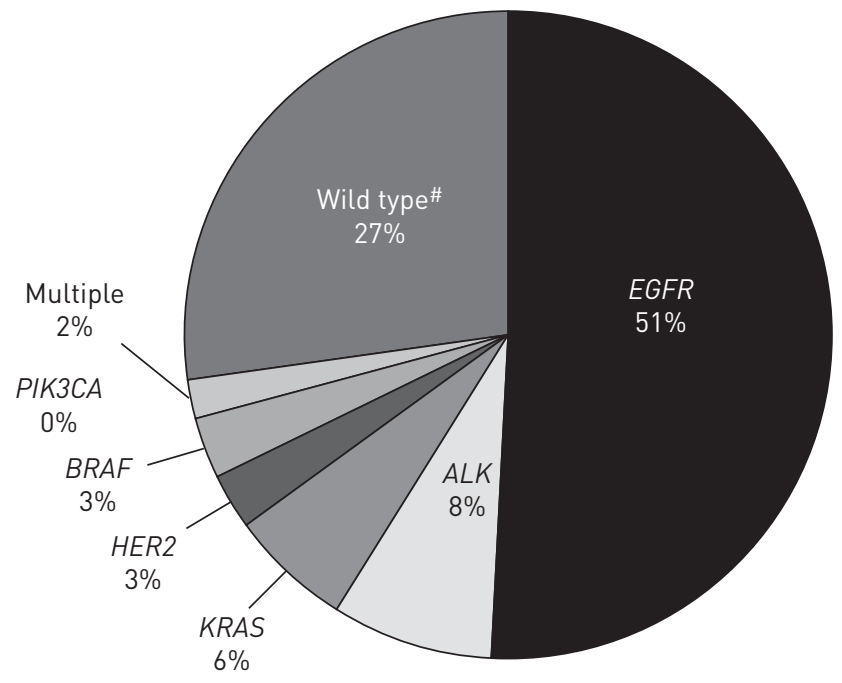

FIGURE 2 Final diagnosis of biomarker analysis in the 284 patients with complete data. ": all biomarkers were wild-type or at least EGFR (epidermal growth factor receptor) and KRAS (Kirsten rat sarcoma viral oncogene homologue) and $A L K$ (anaplastic lymphoma kinase) were wild-type. Double EGFR mutations are categorised under the EGFR category; missing data not shown.

and reporting biases (by using a standardised questionnaire with only two trained, dedicated interviewers), as well as memorisation bias (by delivering the questionnaire prior to the interview to provide more time to answer all questions, and by allowing post-interview revisions and additions). However, our study remains submitted to recall bias. The third critical point is our modest accrual. Although our cohort is one of the largest in this specific field, comparative subgroup analysis was only based on small-number subsets. Therefore, our study lacked sufficient power for estimating differences between patient subsets. For instance, with only 65 patients in the male subgroup, we were able to detect a $23 \%$ difference with $80 \%$ power and a 0.05 alpha risk (using a bilateral test) [23]. This point is especially critical for mutations since all, except EGFR, deal with small numbers. In addition, our study emphasised exposure patterns that have occurred over the last decades, and it thus did not reflect current occupational and social changes that may result in distinct exposure profiles. Finally, our analysis is restricted to biomarkers tested in the routine setting in France. Thus, some important biomarkers commonly expressed in never smokers such as ROS1 (6\% in never smokers) and RET rearrangements were not included in this study [24, 25].

In spite of these limitations, our study carries some strength. First, the never-smoker inclusion criterion was very strict although consensual (fewer than 100 cigarettes in the lifetime) and was checked by physician at inclusion and by staff during interview. Indeed, many studies may be biased, by assessing the "non-smoker" status retrospectively from medical charts, with a risk of overlapping between former- and never-smoker. We also checked for alternative smoking and can thus ensure the lack of contamination from any former (or even very light) smokers. Another noteworthy strength is our comprehensive approach. We collected data on possible exposures to most known or supposed risk factors (including a detailed investigation of occupational exposure), as well as medical data and comprehensive information from tumour biomarker analysis.

This paper reports descriptive finding of the large cohort of European LCINS. Based on these data, we will provide in future papers the main differences in clinical and molecular epidemiology regarding exposure to main risk factors such as passive-smoking, occupational exposure, domestic pollution or reproductive factors [26]. In addition, some new data will result from collaboration with French national institutions for assessment of radon exposure and atmospheric pollution exposure into the BioCAST cohort. Lastly, the population-attributable fraction for most known risk factors of LCINS will be computed taking all this data into account [27].

In conclusion, this study, the largest and most comprehensive analysis of LCINS in a European population, reports differences in age and carcinogen exposure distribution by gender, and emphasizes the crucial role of targetable somatic mutations in this specific population. Physicians facing a never-smoker with a lung cancer should keep in mind than two-thirds of men underwent an occupational exposure and that three-quarters of patients may carry a targetable mutation. The BioCAST/IFCT-1002 study is also an interesting tool for investigating biomarker profiles according to numerous risk exposures and further results are expected soon.

\section{Acknowledgements}

The authors thank M. William Lebossé (Junior Research Assistant), who performed interviews with patients; M. Quan Tran and Antoine Deroy (Data Manager); Pascale Missy (PhD), (all IFCT, Paris, France) who provided 
administrative support; each investigator in the 75 BioCAST participating centres; the patients and their families, who greatly contributed to this work by giving their time to prepare the questionnaire and participate in the interview.

The collaborators of the BioCAST/IFCT-1002 study were: Pierre-Jean Souquet, Hospices Civils de Lyon, Hôpital Lyon Sud, Lyon; Radj Gervais, Centre François Baclesse, Caen; Hélène Doubre, Hôpital Foch, Suresnes, Paris; Eric Pichon, Centre Hospitalier Universitaire de Tours, Tours; Adrien Dixmier, Centre Hospitalier d'Orléans, Orléans; Isabelle Monnet, Centre Hospitalier Intercommunal de Créteil, Paris; Bénédicte Mastroianni, Hospices Civils de Lyon, Hôpital Louis Pradel, Lyon; Michel Vincent, Hôpital Saint-Joseph, Lyon; Jean Tredaniel, Hôpital Saint Joseph, Paris; Marielle Perrichon, Centre Hospitalier de Bourg-en-Bresse, Bourg-en-Bresse; Pascal Foucher, Centre Hospitalier Universitaire Bocage, Dijon; Bruno Coudert, Centre Georges-François Leclerc, Dijon; Denis Moro-Sibilot, Centre Hospitalier Universitaire de Grenoble, Grenoble; Eric Dansin, Centre Oscar Lambret, Lille; Patrick Dumont, Centre Hospitalier de Chauny, Chauny; Lionel Moreau, Centre Hospitalier de Colmar, Colmar; Didier Debieuvre, Centre Hospitalier de Mulhouse, Mulhouse; Jacques Margery, Hôpital d'Instruction des Armées de Percy, Clamart, Paris; Élisabeth Quoix, Centre Hospitalier Universitaire de Strasbourg, Nouvel Hôpital Civil, Strasbourg; Bernard Duvert, Centre Hospitalier de Montélimar, Montélimar; Laurent Cellerin, Centre Hospitalier Universitaire de Nantes, Hôpital Nord Laennec, Nantes; Nathalie Baize, Centre Hospitalier Universitaire d'Angers, Angers; Bruno Taviot, Centre Médical Nicolas de Pontoux, Chalon-sur-Saône; Marie Coudurier, Centre Hospitalier Chambéry, Chambéry; Jacques Cadranel, Assistance PubliqueHôpitaux de Paris, Hôpital Tenon, Paris; Patrick Chatellain, Centre Hospitalier d'Annemasse, Annemasse; Jérôme Virally, Centre Hospitalier Intercommunal d'Aulnay-Sous-Bois, Paris; Virginie Westeel, Centre Hospitalier Universitaire de Besançon, Besançon; Sylvie Labrune, Assistance Publique-Hôpitaux de Paris, Hôpital Ambroise Paré, Boulogne, Boulogne; Laureline le Maignan de Kerangat, Centre Hospitalier Général Le Mans, Le Mans; Jean-Marc Dot, Hôpital d'Instruction des Armées Desgenettes, Lyon; Sébastien Larive, Centre Hospitalier de Mâcon, Mâcon; Christos Chouaid, Assistance Publique-Hôpitaux de Paris, Hôpital Saint-Antoine, Paris; Daniel Coëtmeur, Centre Hospitalier Général de Saint-Brieuc, Saint-Brieuc; Clarisse Audigier-Valette, Centre Hospitalier Intercommunal de Toulon, Toulon; Jean-Pierre Gury, Centre Hospitalier Intercommunal de Vesoul, Vesoul; Luc Odier, Centre Hospitalier de Villefranche sur Saône, Villefranche sur Saône; Tsellina Desfemmes-Baleyte, Centre Hospitalier Universitaire de Caen, Caen; Yannick Duval, Centre Hospitalier de Cannes, Cannes; Patrick Merle, Centre Hospitalier Universitaire de Clermont-Ferrand, Clermont-Ferrand; Gilles Devouassoux, Hospices Civils de Lyon, Hôpital de la Croix Rousse, Lyon; Reza Azarian, Centre Hospitalier de Versailles, Versailles; Patricia Barre, Centre Hospitalier de Cahors, Cahors; Olivier Raffy, Centre Hospitalier de Chartres, Chartres; Philippe Masson, Centre Hospitalier de Cholet, Cholet; Stéphanie Dehette, Centre Hospitalier de Compiègne, Compiègne; Caroline Toussaint Batbedat, Centre Hospitalier de Lagny-sur-Marne, Paris; Gérard Oliviero, Centre Hospitalier de Longjumeau, Paris; Marc Derollez, Polyclinique du Parc, Maubeuge; Nadine Paillot, Centre Hospitalier Régional de Metz, Metz; Jérôme Dauba, Centre Hospitalier de Mont De Marsan, Mont De Marsan; Dominique Herman, Centre Hospitalier de Nevers, Nevers; Jean-Michel Rodier, Assistance Publique-Hôpitaux de Paris, Hôpital Bichat, Paris; Suzanna Bota, Centre Hospitalier Universitaire de Rouen, Rouen; Philippe Brun, Centre Hospitalier de Valence, Valence; Geneviève Letanche, Clinique de Vénissieux Lyon; Mohamed Khomsi, Centre Hospitalier d'Annonay, Annonay; Béatrice Gentil-Lepecq, Centre Hospitalier de Bourgoin-Jallieu, Bourgoin-Jallieu; Philippe Ravier, Cabinet de Pneumologie, Dijon, Dijon; Yassine Hammou, Clinique Mutualiste, Lyon; Fabrice Barlesi, Assistance Publique-Hôpitaux de Marseille, Hôpital Nord, Marseille; Hélène Laize, Centre Hospitalier de Rambouillet, Rambouillet; Pierre Fournel, Institut de Cancérologie de la Loire, Saint-Priest en Jarez; Christelle Clement-Duchene, Centre Hospitalier Universitaire de Nancy, Vandoeuvre-Les-Nancy; Joël Castelli, Centre Hospitalier Départemental Castelluccio, Ajaccio; Sophie Schneider, Centre Hospitalier de Bayonne, Bayonne; Antoine Levy, Centre Hospitalier Jacques Cour, Bourges; Jérôme Dauba, Centre Hospitalier de Dax, Dax; Geneviève Jolimoy, Centre d'Oncologie et de Radiothérapie du Parc, Dijon; Hervé Pegliasco, Fondation Hôpital Ambroise Paré, Marseille; Michel Poudenx, Centre Antoine Lacassagne, Nice; Alain Prevost, Institut Jean-Godinot, Reims; Philippe Romand, Centre Hospitalier de Thonon-les-Bains, Thonon-les-Bains; Laurence Bigay-Game, Centre Hospitalier Universitaire de Toulouse, Toulouse; Etienne Suc, Clinique St Jean Languedoc, Toulouse; all France.

\section{References}

1 Couraud S, Zalcman G, Milleron B, et al. Lung cancer in never smokers: a review. Eur J Cancer 2012; 48: 1299-1311.

2 Sun S, Schiller JH, Gazdar AF. Lung cancer in never smokers: a different disease. Nat Rev Cancer 2007; 7: 778-790.

3 Parkin DM, Bray F, Ferlay J, et al. Global cancer statistics, 2002. CA Cancer J Clin 2005; 55: 74-108.

4 Samet JM, Avila-Tang E, Boffetta P, et al. Lung cancer in never smokers: clinical epidemiology and environmental risk factors. Clin Cancer Res 2009; 15: 5626-5645.

5 Mounawar M, Mukeria A, Le Calvez F, et al. Patterns of EGFR, HER2, TP53, and KRAS mutations of p14arf expression in non-small cell lung cancers in relation to smoking history. Cancer Res 2007; 67: 5667-5672.

6 Mazières J, Peters S, Lepage B, et al. Lung cancer that harbors a HER2 mutation: epidemiologic characteristics and therapeutic perspectives. J Clin Oncol 2013; 31; 1997-2003.

7 Paik PK, Arcila ME, Fara M, et al. Clinical characteristics of patients with lung adenocarcinomas harboring BRAF mutations. J Clin Oncol 2011; 29: 2046-2051.

8 Shaw AT, Yeap BY, Mino-Kenudson M, et al. Clinical features and outcome of patients with non-small-cell lung cancer who harbor EML4-ALK. J Clin Oncol 2009; 27: 4247-4253.

9 Couraud S, Labonne S, Missy P, et al. BioCAST: le Bio-observatoire national du cancer bronchiques chez les patients non fumeurs (IFCT1002). [Lung cancer in never smokers: a French national cohort (BioCAST/ IFCT-1002)]. Rev Mal Respir 2013; 30; 576-583.

10 Bourgkard E, Wild P, Gonzalez $\mathrm{M}$, et al. Comparison of exposure assessment methods in a lung cancer case-control study: performance of a lifelong task-based questionnaire for asbestos and PAHs. Occup Environ Med. 2013; 70; 884-891

11 Nowak F, Soria JC, Calvo F. Tumour molecular profiling for deciding therapy-the French initiative. Nat Rev Clin Oncol 2012; 9: 479-486. 
12 Beau-Faller M, Degeorges A, Rolland E, et al. Cross-validation study for epidermal growth factor receptor and KRAS mutation detection in 74 blinded non-small cell lung carcinoma samples: a total of 5550 exons sequenced by 15 molecular French laboratories (evaluation of the EGFR mutation status for the administration of EGFR-TKIs in non-small cell lung carcinoma (ERMETIC) project: part 1). J Thorac Oncol 2011; 6: 1006-1015.

13 Beau-Faller M, Blons $\mathrm{H}$, Domerg C, et al. A multicenter blinded study evaluating EGFR and KRAS mutation testing methods in the clinical non-small cell lung cancer setting: IFCT/ERMETIC2 Project Part 1: Comparison of testing methods in 20 French molecular genetic National Cancer Institute platforms. J Mol Diagn 2014; 16: 45-55.

14 Coëtmeur D, Briens E, Perrin C, et al. Lung cancer characteristics in 762 never- and 6,246 ever-smoker patients: Study KBP-2010-CPHG. J Clin Oncol 2014; 32: Suppl. 15, 1582.

15 Vineis P, Airoldi L, Veglia F, et al. Environmental tobacco smoke and risk of respiratory cancer and chronic obstructive pulmonary disease in former smokers and never smokers in the EPIC prospective study. BMJ 2005; 330: 277.

16 Kim HR, Shim HS, Chung JH, et al. Distinct clinical features and outcomes in never-smokers with nonsmall cell lung cancer who harbor EGFR or KRAS mutations or ALK rearrangement. Cancer 2012; 118: 729-739.

17 Kawaguchi T, Ando M, Kubo A, et al. Long exposure of environmental tobacco smoke associated with activating EGFR mutations in never-smokers with non-small cell lung cancer. Clin Cancer Res 2011; 17: 39-45.

18 Clément-Duchêne C, Vignaud JM, Stoufflet A, et al. Characteristics of never smoker lung cancer including environmental and occupational risk factors. Lung Cancer 2010; 67: 144-150.

19 Lissowska J, Bardin-Mikolajczak A, Fletcher T, et al. Lung cancer and indoor pollution from heating and cooking with solid fuels: the IARC international multicentre case-control study in Eastern/Central Europe and the United Kingdom. Am J Epidemiol 2005; 162: 326-333.

20 Sun Y, Ren Y, Fang Z, et al. Lung adenocarcinoma from East Asian never-smokers is a disease largely defined by targetable oncogenic mutant kinases. J Clin Oncol 2010; 28: 4616-4620.

21 Paik PK, Johnson ML, D'Angelo SP, et al. Driver mutations determine survival in smokers and never-smokers with stage IIIB/IV lung adenocarcinomas. Cancer 2012; 118: 5840-5847.

22 Barlesi F, Blons H, Beau-Faller M, et al. Biomarkers (BM) France: Results of routine EGFR, HER2, KRAS, BRAF, PI3KCA mutations detection and EML4-ALK gene fusion assessment on the first 10,000 non-small cell lung cancer (NSCLC) patients (pts). J Clin Oncol 2013; 31: Suppl. 15, 8000.

23 Machin D, Campbell MJ, Tan S-B, et al. Sample size tables for clinical studies. 3rd Edn. Hoboken, Wiley-Blackwell, 2008.

24 Lee SE, Lee B, Hong M, et al. Comprehensive analysis of RET and ROS1 rearrangement in lung adenocarcinoma. Mod Pathol 2014; [in press DOI: 10.1038/modpathol.2014.107].

25 Kim HR, Lim SM, Kim HJ, et al. The frequency and impact of ROS1 rearrangement on clinical outcomes in never smokers with lung adenocarcinoma. Ann Oncol 2013; 24: 2364-2370.

26 Couraud S, Dumont P, Moreau L, et al. Impact of Passive Smoking on molecular pattern in Never Smokers with Non-Small Cell Lung Cancer: Findings from the BioCAST / IFCT-1002 Study. Abstract\#O18.04. J Thorac Oncol 2013; 8: Suppl. 2, S1-S1410.

27 Sisti J, Boffetta P. What proportion of lung cancer in never-smokers can be attributed to known risk factors? Int J Cancer 2012; 131: 265-275. 